\title{
A DICE PROBABILITY PROBLEM
}

\author{
P. McMULLEN
}

Abstract. Two different approaches to a probability problem involving convex polytopes lead to a geometric proof of an integral geometric result about mixed surface areas. The proof can be modified to cover the corresponding results about mixed volumes.

1. Introduction. W. J. Firey has informally posed the following problem: What is the most probable way that two dice which encounter each other randomly will meet? As we shall see, the only ways they can meet with positive probability are vertex against face or edge against edge; the latter turns out to be more likely.

The main interest of this paper, however, is not in the solution of this problem, but in the fact that there are two approaches to the solution. The first is integral geometric, and the second is more directly geometric. When we compare these approaches, we find that we have a geometric proof of a fairly important integral geometric formula involving the mixed surface areas of convex bodies. A slight modification of the argument proves the similar formula for mixed volumes.

2. Formulation of the problem. We consider the following general problem. Let $P$ and $Q$ be $n$-polytopes ( $n$-dimensional convex polytopes) in $E^{n}$, and let $\Gamma$ be a rigid motion of $E^{n}$ (say $x \Gamma=x \Phi+t$, where $\Phi \in S O_{n}$ is a rotation, and $t \in E^{n}$ is a translation vector), such that $P$ and $Q \Gamma$ touch. (Thus we are considering only the relative positions of $P$ and $Q$.) We see that this occurs precisely when

$$
t \in b d(P-Q \Phi) \text {. }
$$

For, since $P \cap Q \Gamma \neq \varnothing$, we have $t \in P-Q \Phi$. However, since int $P \cap$ int $Q \Gamma=\varnothing$, $P$ and $Q \Gamma$ are separated by a hyperplane, with normal $u$ say, and their support functions satisfy

Hence

$$
h(P, u)=-h(Q \Gamma,-u)=-h(Q \Phi+t,-u) .
$$

$$
\langle t, u\rangle=h(P, u)+h(-Q \Phi, u)=h(P-Q \Phi, u),
$$

so that $t \in b d(P-Q \Phi)$, as claimed.

This suggests that the appropriate measure to use is $\iint d t d \Phi$, where $d t$ is the $(n-1)$-dimensional surface area measure (with $t \in b d(P-Q \Phi)$ ), and $d \Phi$ is the invariant (Haar) measure on $S O_{n}$ (normalized, say, so that the total measure of $S O_{n}$ is 1). Notice that this measure is rigid motion invariant, in the sense that if $\Delta$ is any fixed rigid motion, and $\mathscr{G}$ is any set of rigid motions $\Gamma$ such that $P$ and $Q \Gamma$ touch, then the same measure is assigned to $\mathscr{G}$ and to the corresponding set $\Delta^{-1} \mathscr{G} \Delta$ of rigid motions $\Gamma^{\prime}$ for which $P \Delta$ and $Q \Delta \Gamma^{\prime}$ touch. (In the last section but one, we shall make further remarks, which provide even more justification for using this measure.)

Firey's original problem can now be generalized, and stated in the following form: "Given two $n$-polytopes $P$ and $Q$ in $E^{n}$, what is the measure of the set of rigid motions $\Gamma$ such that $P$ and $Q \Gamma$ touch, a $p$-face of $P$ against a $q$-face of $Q$, for each $p$ and $q$ ?" 
3. The first solution. We first observe that those rotations $\Phi$ for which a facet of $P-Q \Phi$ is the sum of a $p$-face of $P$ and a $q$-face of $-Q \Phi$, for some $p$ and $q$ with $p+q \geqslant n$, form a set of measure 0 in $S O_{n}$. Thus, if $p+q \geqslant n, P$ and $Q \Gamma$ will touch with a $p$-face of $P$ against a $q$-face of $Q \Gamma$ with probability 0 . So, we may henceforth assume that $P$ and $Q \Gamma$ are in relatively general position. In this case, for a fixed rotation $\Phi$, the translations $t$ corresponding to faces of $P-Q \Phi$ of dimension less than $n-1$ also form a set of zero measure. In other words:

LEMMA. The only encounters of $P$ and $Q \Gamma$ with positive probability are where a $p$-face of $P$ meets a $q$-face of $Q$, with $p+q=n-1$.

Now, for fixed $\Phi$, the surface area of $P-Q \Phi$ is

$$
S(P-Q \Phi)=\sum_{p=0}^{n-1}\left(\begin{array}{c}
n-1 \\
p
\end{array}\right) S\left(P^{(p)},(-Q \Phi)^{(n-p-1)}\right)
$$

where $S\left(A^{(a)}, B^{(b)}, \ldots\right)$ means the mixed surface area of $A$ taken $a$ times, $B$ taken $b$ times, and so on. Furthermore, when $P$ and $Q \Gamma$ are in relatively general position, the term $\left(\begin{array}{c}n-1 \\ p\end{array}\right) S\left(P^{(p)},(-Q \Phi)^{(n-p-1)}\right)$ is the total area of those facets of $P-Q \Phi$ which are the sum of a $p$-face of $P$ and an $(n-p-1)$-face of $-Q \Phi$. That is, this term measures the encounters of $P$ and $Q \Gamma$, with a $p$-face of $P$ against an $(n-p-1)$ face of $Q \Gamma$.

This already answers the case of two congruent cubes in $E^{3}$ with which we began. For, we have (if $n=3$ )

$$
S(P,-Q \Phi)^{2} \geqslant S(P) S(-Q \Phi)
$$

[see Bonnesen-Fenchel, 1934, 52]; in fact, we have strict inequality, since $P$ and $-Q \Phi$ are not homothetic, being in relatively general position. Thus, if $S(P)=S(Q)=S(-Q \Phi)$, we have $S(P,-Q \Phi)>S(P)$, and so

$$
2 S(P,-Q \Phi)>S(P)+S(Q) \text {. }
$$

Since the integrals of the left and right sides over $\mathrm{SO}_{3}$ measure, respectively, the edge against edge and vertex against face encounters, we at once conclude:

Theorem 1. Let $P$ and $Q$ be 3-polytopes in $E^{3}$ with the same surface area. If $P$ and $Q$ are randomly placed so that they touch, they are strictly more probable to do so edge against edge than vertex against face.

The general problem can also be solved without difficulty, using a result of Hadwiger [1957, cf. 6.2.4]. For, we have:

THEOREM 2.

$$
\int_{S_{0}} S\left(P^{(p)},(-Q \Phi)^{(n-p-1)}\right) d \Phi=\frac{1}{S(B)} S\left(P^{(p)}, B^{(n-p-1)}\right) S\left(Q^{(n-p-1)}, B^{(p)}\right),
$$

where $B$ is the unit ball in $E^{n}$.

Notice that $S\left(K^{(r)}, B^{(n-r-1)}\right)=n W_{n-r}(K)$, where $W_{n-r}(K)$ is the $(n-r)$-th Quermassintegral of $K$. In particular, in case $n=3, S(K, B)=2 \pi w(K)$, where $w(K)$ is the mean width of $K$. We therefore deduce: 
THEOREM 3. The probabilities that two congruent cubes in $E^{3}$ touching randomly will meet edge against edge and vertex against face are in proportion $3 \pi: 8$.

4. The second solution. The integral geometric formula of the last section, from which the solution to our problem follows, depends upon a characterization (also due to Hadwiger [1957, 6.1.10]) of the Quermassintegrals. In this section we shall use our probabilistic interpretation to point the way to a geometric proof of the formula of Theorem 2.

As before, let $P$ and $Q$ be $n$-polytopes in $E^{n}$. We consider those rigid motions $\Gamma$ of $E^{n}$, such that $P$ and $Q \Gamma$ touch, with a particular $p$-face $F$ of $P$ against a particular $(n-p-1)$-face $G \Gamma$ of $Q \Gamma$. We must be careful to measure these rigid motions in the same way as before; that is, we first measure, for a fixed rotation $\Phi$, the appropriate set of translations $t$ (belonging to $F-G \Phi$ ), and then integrate over $S O_{n}$.

So, let us first fix $\Phi$ (and, again, we assume that $P$ and $Q \Gamma$ are in relatively general position). The possible translations $t$ run through the facet $F-G \Phi$ of $P-Q \Phi$, as we have just remarked, and the area of $F-G \Phi$ is the product of their respective $p$ - and $(n-p-1)$-dimensional volumes $V_{p}(F)$ and $V_{n-p-1}(G)$ by a factor which depends only upon the relative orientation of aff $F$ and aff $(-G \Phi)$ in the hyperplane $H=\operatorname{aff}(F-G \Phi)$. We now integrate over all the rotations $\Phi$. We can split the possible rotations into three stages: rotating the hyperplane $H_{1}=$ aff $(F \cup G \Gamma$ ) parallel to $H$ about $F$ (or aff $F$ ) while still supporting $P$, rotating the $(n-p-1)$-flat aff $G \Gamma$ in $H_{1}$, and rotating $Q \Gamma$ about $G \Gamma$, while ensuring that $H_{1}$ supports $Q \Gamma$. (This factorization is not unique, but the integration we shall now perform is independent of the choice of rotations as factors.) The first and third stages give us terms proportional to the external $(n-p)$ - and $(p+1)$-dimensional angles $\gamma_{n-p}(F, P)$ of $P$ at $F$ and $\gamma_{p+1}(G, Q)$ of $Q$ at $G$, respectively, while the second gives a term depending only on the dimension $p$ (of aff $F$ ), and not on the particular faces $F$ and $G$. That is, the total measure of these rigid motions is

$$
\kappa_{p} V_{p}(F) V_{n-p-1}(G) \gamma_{n-p}(F, P) \gamma_{p+1}(G, Q) \text {. }
$$

Summing over all $p$-faces $F$ of $P$ and $(n-p-1)$-faces $G$ of $Q$ gives us a total of

$$
\kappa_{p}\left\{\sum_{F} \gamma_{n-p}(F, P) V_{p}(F)\right\}\left\{\sum_{G} \gamma_{p+1}(G, Q) V_{n-p-1}(G)\right\}=\lambda_{p} W_{n-p}(P) W_{p+1}(Q) ;
$$

that is, a term proportional to the product of the respective Quermassintegrals $W_{n-p}(P)$ and $W_{p+1}(Q)$. (The above expressions for the Quermassintegrals can be deduced from, for example, Hadwiger [1957, 6.1.8 (48).]) Since we have already evaluated this term as an integral in $\$ 3$, we see that we have found an alternative proof of Theorem 2. (The value of the constant $\lambda_{p}$ can be found by using the continuity of the Quermassintegrals, and substituting $P=Q=B$, the unit ball.)

5. A generalization. The foregoing naturally raises the question of the existence of a direct proof of the most general integral geometric formula of this type (Hadwiger 1957, 6.2.4).

\section{THEOREM 4.}

$$
\int_{S_{O_{n}}} V\left(P^{(r)},(Q \Phi)^{(n-r)}\right) d \Phi=\frac{1}{V(B)} W_{n-r}(P) W_{r}(Q) .
$$


To construct such a proof, we modify slightly the argument of the previous section. As we saw, the first stage of measuring the rotations involved rotating a supporting hyperplane $H_{1}$ about a $p$-face $F$. That is, to within a constant, we evaluated

$$
\int_{\sigma(F, P)} V_{p}(F) d u=\kappa \gamma_{n-p}(F, P) V_{p}(F),
$$

where $\sigma(F, P)$ is the $(n-p-1)$-dimensional spherical image of $F$, the unit vector $u$ (normal to $H_{1}$ ) varies over $\sigma(F, P)$, and the measure is the usual spherical $(n-p-1)$-dimensional measure. Suppose, instead, we multiply by the support function $h(P, u)$ of $P$ before integrating. Summing over all $p$-faces $F$ of $P$, the corresponding term involving $P$ is now

$$
\sum_{F} \int_{\sigma(F, P)} V_{p}(F) h(P, u) d u=\lambda W_{n-p-1}(P),
$$

proportional to the $(n-p-1)$-st Quermassintegral of $P$.

On the other hand, the integrand on the left side in the statement of Theorem 2 is transformed into

$$
\sum h(P, u) S(F-G \Phi)=\mu V\left(P^{(p+1)},(-Q \Phi)^{(n-p-1}\right),
$$

where the sum is taken. over the (finitely many) facets $F-G \Phi$ (with $\operatorname{dim} F=p$, $\operatorname{dim} G=n-p-1$ ) of $P-Q \Phi$, and $u$ is the corresponding normal vector. If we now put $r=p+1$ and replace $Q$ by $-Q$, we obtain Theorem 4. (As before, we evaluate the constant of proportionality by setting $P=Q=B$.)

6. Remarks. The results of $\S \S 4$ and 5 (that is, the geometric proofs of Theorems 2 and 4) are clearly independent of their probabilistic interpretation in Theorems $l$ and 3 . So, to this extent, the possibly arbitrary choice of our probability measure is irrelevant. However, the fact that the measure is rigid motion invariant would seem to justify the choice, and in this section we shall produce further evidence in its favour.

We argue in the spirit of Firey [1972]. Suppose, instead of demanding that $P$ and $Q \Gamma$ touch, we require that the (euclidean) distance between them be at most some positive number $\eta$. This time, for a fixed rotation $\Phi$, the translations $t$ run through the shell

$$
((P-Q \Phi)+\eta B) \backslash \operatorname{int}(P-Q \Phi)
$$

The volume of this shell is

$$
\eta S(P-Q \Phi)+O\left(\eta^{2}\right)
$$

Moreover, if $P$ and $Q \Gamma$ are in relatively general position, the term

$$
\eta\left(\begin{array}{c}
n-1 \\
p
\end{array}\right) S\left(P^{(p)},(-Q \Phi)^{(n-p-1)}\right)
$$

corresponds to the case when the distance between $P$ and $Q \Gamma$ is attained by a point in a $p$-face of $P$ and a point in an $(n-p-1)$-face of $Q \Gamma$. If we divide through by $\eta$, we conclude that the relative measures (which are now positive) of all such rigid 
motions are proportional to

$$
\left(\begin{array}{c}
n-1 \\
p
\end{array}\right) \int_{S_{0}} S\left(P^{(p)},(-Q \Phi)^{(n-p-1)}\right) d \Phi,
$$

with a remainder term $O(\eta)$ corresponding to the cases where the closest points of $P$ and $Q \Gamma$ belong to faces whose dimensions sum to less than $n-1$. As we allow $\eta$ to tend to zero (and so the closest points to tend to coincidence), we obtain the probabilistic measure introduced in $\$ 2$.

7. An alternative problem. Professor C. A. Rogers, on reading the foregoing, suggested the following alternative problem, which in some ways represents a more realistic physical situation. We take the point of view of, say, a nuclear physicist. One of our polytopes $Q$, with a particular orientation $\Theta$, is designated as a target, and the other $P$, oriented by $\Psi$, is projected towards $Q$ in a fixed direction $u$. We ask what proportion of all their encounters occur with a $p$-face of $P$ against a $q$-face of $Q$.

Surprisingly, perhaps, the answer is the same as that to our original problem. For, we vary our shots by means of a translation vector $v$ orthogonal to $u$; we shall then have an encounter, if, and only if,

$$
v \in(P \Psi-Q \Theta) \Pi_{u},
$$

where $\Pi_{u}$ denotes orthogonal projection in direction $u$. If we make our usual assumption that $P \Psi$ and $Q \Theta$ are in relatively general position, then the point of contact for a particular such $v$ will be $v+\lambda u$, where

$$
\lambda=\max \{\mu \mid v+\mu u \in P \Psi-Q \Theta\} \text {. }
$$

Thus those encounters with a $p$-face of $P$ against a $q$-face of $Q$ correspond to points in faces $F \Psi-G \Theta(\operatorname{dim} F=p, \operatorname{dim} G=q)$ of $P \Psi-Q \Theta$ visible from direction $u$. Moreover, the measure of the appropriate translations $v$ is the area of the projection $(F \Psi-G \Theta) \Pi_{u}$ of this face, which is zero unless $p+q=n-1$.

We now apply Cauchy's integral formula [see Hadwiger, 1957, 6.1.5] concerning surface area. Instead of varying $\Psi$ and $\Theta$, we replace $u$ by $u \Psi^{-1}$, and vary $\Psi$, while (for the moment) keeping $\Phi=\Theta \Psi^{-1}$ fixed. We integrate the area of

$$
(P \Psi-Q \Theta) \Pi_{u}=((P-Q \Phi) \Psi) \Pi_{u}
$$

with respect to $\Psi$ over $S O_{n}$, to obtain a constant multiple of $S(P-Q \Phi)$; as before, the term

$$
\left(\begin{array}{c}
n-1 \\
p
\end{array}\right) S\left(p^{(p)},(-Q \Phi)^{(n-p-1)}\right)
$$

corresponds to those encounters with a $p$-face of $P \Psi$ against an $(n-p-1)$-face of $Q \Theta$, and so integrating with respect to $\Theta$ yields us the same relative probabilities as in our original problem.

\section{References}

T. Bonnesen and W. Fenchel. Theorie der konvexen Körper (Springer, Berlin, 1934, reprinted Chelsea, New York, 1948). 
W. J. Firey. "An integral-geometric meaning for lower order area functions of convex bodies", Mathematika, 19 (1972), 205-212.

H. Hadwiger. Vorlesungen ïber Inhalt, Oberfläche und Isoperimetrie (Springer, Berlin-GöttingenHeidelberg, 1957).

University College London,

Gower Street, London WC1E 6BT.
52A25: CONVEX SETS AND GEOMETRIC INEQUALITIES; Convex polyhedra.

60D05: PROBABILITY THEORY; Geometric probability.

Received on the 11th of March, 1974. 